USC-94-10

hep-th/yymmxxx

\title{
Exact solution of a massless scalar field with a relevant boundary interaction
}

\author{
P. Fendley, H. Saleur* and N.P. Warner \\ Department of Physics, University of Southern California \\ Los Angeles CA 90089-0484
}

\begin{abstract}
We solve exactly the "boundary sine-Gordon" system of a massless scalar field $\phi$ with a $\cos \beta \phi / 2$ potential at a boundary. This model has appeared in several contexts, including tunneling between quantum-Hall edge states and in dissipative quantum mechanics. For $\beta^{2}<8 \pi$, this system exhibits a boundary renormalization-group flow from Neumann to Dirichlet boundary conditions. By taking the massless limit of the sine-Gordon model with boundary potential, we find the exact $S$ matrix for particles scattering off the boundary. Using the thermodynamic Bethe ansatz, we calculate the boundary entropy along the entire flow. We show how these particles correspond to wave packets in the classical Klein-Gordon equation, thus giving a more precise explanation of scattering in a massless theory.
\end{abstract}

* Packard Fellow

$6 / 94$ 


\section{Introduction}

Many fundamental models of statistical mechanics can be expressed as $1+1$ dimensional field theories with a boundary. A number of these field theories can be exactly solved by applying the powerful techniques of integrability [1]. Such models include the Kondo problem, a number of models of dissipative quantum mechanics, and the model we treat here: a free boson with a periodic potential on the boundary.

We consider the "boundary sine-Gordon" problem of a massless scalar field $\phi(\sigma)$ on the segment $0<\sigma<L$. The Lagrangian is

$$
\mathcal{L}=\frac{1}{2} \int_{0}^{L} d \sigma\left(\partial_{\mu} \phi\right)^{2}+g \cos \left[\frac{\beta}{2} \phi(0)\right]
$$

This model arises in a number of contexts because one can often map interacting fermions (the Luttinger liquid or massless Thirring model) onto a free boson. Coupling the fermions to a boundary potential, for example, gives this boundary interaction for the bosons [2]. This model has also appeared in dissipative quantum mechanics, where it describes a particle moving in a periodic potential with a frictional force [3].

The model in the bulk (a free massless scalar field) is, of course, a conformal field theory. For $g=0$ and $g \rightarrow \infty$, the combination of left and right conformal symmetries is preserved in the presence of a boundary [4]. However for $\beta^{2}<8 \pi$, the interaction breaks the scale invariance, with the parameter $g$ providing a mass scale. At $g=0$, the boundary dimension of the operator $\cos [\beta \phi / 2]$ is $x=\frac{\beta^{2}}{8 \pi}$. For $0<x<1$ a perturbation by this operator is relevant and induces a renormalization group flow between two different conformal boundary conditions. Our purpose is to study this flow explicitly.

To understand the boundary condition induced by the interaction, we examine the energy-momentum tensor $T_{\mu \nu}=\frac{1}{2} \partial_{\mu} \phi \partial_{\nu} \phi$, which satisfies the current conservation $\partial_{\mu} T_{\mu \nu}=0$. The Hamiltonian is defined as $H=\int_{0}^{L} T_{t t} d \sigma-g \cos [\beta \phi(0) / 2]$. The interaction adds a boundary contribution to $H$, so that the boundary itself can hold energy. Without the boundary interaction, we would require that the component $T_{\sigma t}$ vanish at $\sigma=0$ and $\sigma=L$ to ensure that no energy crosses the boundaries (i.e. $d H / d t=0$ ). Thus in general, energy conservation gives a dynamical boundary condition, requiring that $T_{\sigma t}=\partial_{t} g \cos [\beta \phi / 2]$ at $\sigma=0$, or

$$
\frac{d \phi(0)}{d \sigma}=g \beta \sin \left[\frac{\beta}{2} \phi(0)\right]
$$


This is a classical equation, but the same relation holds in the quantum field theory after a renormalization of parameters [5]. Thus the boundary interaction causes a flow between Neumann boundary conditions $(\partial \phi(0)=0)$ in the UV and Dirichlet $(\phi(0)=4 n \pi / \beta, n$ integer) in the IR. These two boundary conditions are dual to each other.

To solve this model, we shall make use of the powerful constraints of integrability. There are an infinite number of conserved kinematic quantities for the free boson. A generic boundary interaction will destroy them. However, a boundary interaction of the form (1.1) (or equivalently, a boundary condition of the form (1.2)) still preserves an infinite number of appropriately-modified conserved quantites. This can be seen explicitly in perturbation theory and in the classical limit, by taking the massless limit of the sine-Gordon model with a boundary [5].

A particularly useful approach to integrable theories with and without boundaries is to find the exact $S$ matrix of the physical quasiparticles of the theory [6-8]. From the $S$ matrix one can calculate a number of exact thermodynamic quantities such as the free energy, specific heat, susceptibility and boundary entropy. This is done by using the thermodynamic Bethe ansatz [9,10]. We describe this technique in the presence of a boundary in sect. 4 .

To understand the states of our theory, we look at the system far away from the boundary. Here the "bulk" theory is just that of a free massless scalar field. Ordinarily, with a massless bulk theory, one describes the excitations in terms of plane waves (perhaps around some non-trivial topological background). However, this is not a convenient basis to work with once one has included the boundary interaction. The reason for this is simple. The plane waves are no longer eigenstates of the conserved charges, so a plane wave scattering off the boundary could result in a possibly-horrible combination of other plane waves.

A basis where the states have well-defined eigenvalues of the conserved charges is given by the massless limit of sine-Gordon solitons [11]. In other words, we consider therefore the problem given by the Lagrangian (1.1) as the $G \rightarrow 0$ limit of a problem which is massive in the bulk, with perturbation $\delta \mathcal{L}=-G \int_{0}^{L} d \sigma \cos \beta \phi$. With this particular combination of bulk and boundary perturbations (notice the $\beta$ in the bulk and the $\beta / 2$ on the boundary) the model is integrable [5]. In sect. 2, we will make this change-of-basis explicit by showing that the massless solitons obey the classical equation (1.2), and that they retain their form after scattering. 
When one changes basis from the free plane waves to the massless sine-Gordon solitons, the scattering of the bulk particles is no longer trivial or even diagonal. We describe such processes by massless scattering [12,13,11]. The integrability of the model is crucial to such a description. In a basis with well-defined charges, all collisions must be completely elastic, so that momenta are conserved individually and particle production is impossible. Even with this crucial simplification, it is still not immediately obvious what it means to scatter two particles traveling in the same direction at the speed of light. There are several ways to define a bulk $S$ matrix in the integrable models we are studying. One is as the phase which results when commuting two particle-creation operators (the ZamolodchikovFaddeev algebra). This phase is equivalently realized as a matching condition on a twoparticle wavefunction $\psi\left(\sigma_{1}, \sigma_{2}\right)$ between the regions $\sigma_{1} \ll \sigma_{2}$ and $\sigma_{1} \gg \sigma_{2}$. One can explicitly compute this phase in a lattice Bethe ansatz by forming a two-particle state and bringing one of the two particles around the periodic world, i.e. sending $\sigma_{1} \rightarrow \sigma_{1}+L$, where space is a circle of circumference $L$ [14]. In other words, one studies the monodromy properties of massless particles [15].

We emphasize that by "massless" limit, we mean that the quasiparticles are massless (they have the dispersion relation $E= \pm P$ ). The theory is not scale-invariant because of the boundary interaction. With this scale (we call it $T_{B}$ ), boundary scattering can depend on the momentum of the incident particle, because there exists a dimensionless parameter $P / T_{B}$. Similarly, thermodynamic quantities like the free energy depend on the ratio $T / T_{B}$.

The outline of this paper is as follows. In sect. 2, we discuss the classical limit of the problem in order to give a more precise understanding of massless scattering. While it is conceptually important, the details are not required for what follows. In sect. 3, we give the exact quantum $S$ matrix by taking the massless limit of the boundary $S$ matrix for the sine-Gordon model [5]. We show that it has all of the desired properties, and that it agrees with a previously-derived result at $\beta^{2}=4 \pi$ [16]. In sect. 4 , we use the $S$ matrix to derive the boundary free energy, thus showing how the boundary entropy flows between the Neumann and Dirichlet fixed points. In sect. 5 we present some thoughts on future directions.

\section{Understanding massless scattering via the classical limit}

Studying the scattering of massless particles has given a wealth of useful information generally unavailable by other means; for example, as we discuss in sect. 4 , it enables the 
calculation of the exact free energy (c-function) flowing between two fixed points (for a review, see [11]). One subtlety is that in such a description there can be non-trivial bulk scattering even if a massless theory is linear and obeys superposition, and thus appears to have trivial monodromy. For example, in the Kondo problem, the bulk model consists of free massless electrons; in this paper we discuss a free massless scalar field. The reason for the non-trivial scattering, as indicated in the introduction, is that when coupling to a boundary, it is much more useful to make a (non-linear) change of basis of particles. The advantage is that boundary scattering is much easier to describe; the added complication is that the scattering in the bulk is no longer trivial. Thus in the multi-channel Kondo problem, the appropriate quasiparticles are kinks in a potential with mutiple degenerate wells [17], whereas in this paper they are kinks (and breathers) in a potential with an infinite number of wells. This change-of-basis is often not very explicit, since it can be deduced indirectly from the Bethe ansatz solution, or inferred from demanding the appropriate symmetry structure. Our purpose in this section is to elucidate massless scattering and hopefully give some physical insight by describing the classical limit of an important example.

We consider here a classical scalar field $\phi(\sigma, t)$ satisfying the Klein-Gordon equation:

$$
\partial_{t}^{2} \phi-\partial_{\sigma}^{2} \phi=0
$$

A dimensional parameter $g$ is introduced by requiring that theory live on the half-line $[0, \infty)$, with boundary conditions:

$$
\left.\partial_{\sigma} \phi\right|_{\sigma=0}=\left.g \sin \left(\frac{1}{2}\left(\phi-\phi_{0}\right)\right)\right|_{\sigma=0}
$$

for some constants $g$ and $\phi_{0}$. (Classically, one can scale out the parameter $\beta$ in (1.2).)

\subsection{The massless limit of sine-Gordon}

In many massless scattering problems an intrinsic bulk massive perturbation has been implicitly or explicitly introduced, and then set to zero. Moreover, the perturbation is always chosen so that the theory remains integrable off the critical point. The fact that one is considering a massless limit of a massive integrable model leads one to a preferred basis of 'wave packets' or 'wavelets' for the massless theory. This enables one to give a particle interpretation to the massless theory, and defines the concept of massless scattering. A given massless theory can, of course, be the limit of several massive integrable theories 
(e.g. the Ising model with a magnetic or energy perturbation), and as a result there can be several different massless scattering matrices, leading to distinct descriptions of the massless theory (see, for example, [18]). A bulk mass can be introduced to our example by considering (2.1) to be the $m \rightarrow 0$ limit of the sine-Gordon equation:

$$
\partial_{t}^{2} \phi-\partial_{\sigma}^{2} \phi=-m^{2} \sin (\phi)
$$

This massive model was shown to be integrable in the presence of the boundary condition (2.2) in [5].

For either of these partial differential equations we want to consider wave packets of finite energy (which means that $\phi$ must approach a constant value suitably fast as $\sigma \rightarrow \pm \infty)$. For any non-linear, integrable, partial differential equation, there are infinitely many conserved quantities, and in the limit in which the mass vanishes, these conserved quantities become conserved quantities for the massless theory. For the sine-Gordon theory, the massless limit of these conserved quantities are special polynomials in the partial derivatives of $\phi$. The most general characterization of the preferred wave packets in the massless theory are those wave packets that are eigenfunctions of the massless limit of these conserved quantities. An equivalent characterization of these massless wave packets is to take the non-dispersive wave packets of the massive theories and take an appropriate limit in which their energy remains finite when $m \rightarrow 0$.

There are two types of finite-energy solutions of the classical sine-Gordon equation (2.3): solitons, which are time-independent and topologically non-trivial, and breathers, which are time-dependent and topologically trivial. Intuitively, a breather can be thought of as a bound state of a kink and an antikink oscillating in and out (i.e. breathing). In this section, we will discuss only the solitons; the analysis for the breathers follows analogously.

A major triumph of the theory of non-linear partial differential equations was the construction of explicit solutions of (2.3) for any number of moving solitons (see, for example, [19]). The solitons' energies and momenta are conveniently expressed in terms of rapidities $\alpha_{j}$, defined by $E_{j}=m \cosh \alpha_{j}$ and $P_{j}=m \sinh \alpha_{j}$. The velocity of each is thus given by $\tanh \alpha_{j}$ (positive for a right-moving soliton). We have set the speed of "light" to be 1.

To find the scattering of two solitons in the massless limit, consider a two-soliton solution of 2.3$)$ on $(-\infty, \infty)$. This solution is usually expressed as:

$$
\phi(\sigma, t)=4 \arg (\tau) \equiv 4 \arctan \left(\frac{\mathcal{I} m(\tau)}{\mathcal{R} e(\tau)}\right)
$$


where the $\tau$-function solution is given by:

$$
\begin{aligned}
\tau=1 & -\epsilon_{1} \epsilon_{2}\left(\tanh \frac{\alpha_{1}-\alpha_{2}}{2}\right)^{2} e^{-E_{1}(\sigma-a)-E_{2}(\sigma-b)+P_{1} t+P_{2} t} \\
& +i\left\{\epsilon_{1} e^{-E_{1}(\sigma-a)+P_{1} t}+\epsilon_{2} e^{-E_{2}(\sigma-b)+P_{2} t}\right\}
\end{aligned}
$$

The constants $a$ and $b$ represent the initial positions of the two solitons, and $\epsilon_{j}=+1$ if the $j^{\text {th }}$ soliton is a kink, while $\epsilon_{j}=-1$ if it is an anti-kink.

For a wavepacket to have finite energy in the massless limit $m \rightarrow 0$, the rapidity $|\alpha|$ must go to infinity. We thus define $\alpha \equiv \Lambda+\theta$, and let $\Lambda \rightarrow \infty$ such that the parameter $\mu \equiv \frac{1}{2} m e^{\Lambda}$ remains finite. The energy and momentum of a right-moving "massless" soliton then reads $E=P=\mu e^{\theta}$. For a left mover, $\alpha \equiv-\Lambda+\theta$, and its energy and momentum read $E=-P=\mu e^{-\theta}$.

Suppose that both of these solitons are right-moving. Then the massless limit yields:

$$
\tau=1-\epsilon_{1} \epsilon_{2} e^{-\Delta} e^{-E_{1}(\eta-a)-E_{2}(\eta-b)}+i\left\{\epsilon_{1} e^{-E_{1}(\eta-a)}+\epsilon_{2} e^{-E_{2}(\eta-b)}\right\}
$$

where $\eta=(\sigma-t)$ and

$$
\Delta \equiv-\log \left[\left(\tanh \left(\theta_{1}-\theta_{2}\right)\right)^{2}\right]
$$

This is manifestly a solution of the Klein-Gordon equation, and by construction is an eigenfunction of all of the conserved quantities of the massless limit of the sine-Gordon equation. (It is shown in [11] that it remains an eigenfunction after quantization - the expectation values $\left\langle E^{2 k-1}\right\rangle$ are precisely the quantum conserved charges derived in [20].) It is almost a superposition of two single-soliton wave packets. Observe that:

$$
\begin{gathered}
\arg \left[1-\epsilon_{1} \epsilon_{2} e^{-E_{1}(\eta-a)-E_{2}(\eta-b)}+i\left\{\epsilon_{1} e^{-E_{1}(\eta-a)}+\epsilon_{2} e^{-E_{2}(\eta-b)}\right\}\right] \\
=\arg \left[1+i \epsilon_{1} e^{-E_{1}(\eta-a)}\right]+\arg \left[1+i \epsilon_{2} e^{-E_{2}(\eta-b)}\right]
\end{gathered}
$$

The factor $\Delta$ thus measures the extent to which the two-soliton solution is not a superposition of one-soliton solutions.

More precisely, consider the limit $a \rightarrow \infty, \eta \rightarrow \infty$ so that $E_{1}(\eta-a)$ is finite. This corresponds to moving the first kink off to $\sigma=+\infty$ and following it. The $\tau$ function collapses to the one-kink form $\tau=1+i \epsilon_{1} e^{-E_{1}(\eta-a)}$. Moving this soliton through the second one corresponds to taking it to $\sigma=-\infty$, or taking the limit $a \rightarrow-\infty, \eta \rightarrow-\infty$ (with $E_{1}(\eta-a)$ finite). Discarding an overall multiplicative factor (which is irrelevant in the computation of $\phi=4 \arg (\tau))$, we see that in this limit, $\tau=1+i \epsilon_{1} e^{-E_{1}(\eta-a)-\Delta}$. Thus 
these preferred Klein-Gordon wave packets exhibit non-trivial monodromy. The foregoing time delay $\Delta$ is precisely the classical form of a massless scattering matrix. One can then semi-classically quantize this [21,22]; the analysis is identical in massive and massless cases, because the difference $\theta_{1}-\theta_{2}$ is the same in both cases. We will bypass this step because the exact quantum answer is already known [6].

One obtains the same $\Delta$ for two left-moving solitons. For a left-moving and a right-

moving soliton colliding one easily sees that the massless limit of $\left(\tanh \frac{1}{2}\left(\alpha_{1}-\alpha_{2}\right)\right)^{2}$ is unity. The solution collapses to the superposition of a left-moving wave packet and a right-moving wave packet exactly as in (2.7), with no time delay. This is the classical manifestation of the fact that the left-right quantum scattering matrix $S_{L R}$ elements are at most rapidity-independent phase shifts.

\subsection{Klein-Gordon with a non-linear integrable boundary condition}

Consider now the Klein-Gordon equation on $[0, \infty)$ with the boundary condition (2.2). The non-linear boundary conditions destroy the naive conserved quantities of the KleinGordon equation. However, by reversing the argument in the appendix of [5] one finds that the system will still have higher-spin conserved quantities, namely those of the massive sineGordon system. Thus the bulk theory is Klein-Gordon, but in this instance there are a preferred set of wave packets that scatter from the boundary without dispersion. These wave packets are once again the massless limit of the sine-Gordon solitons.

A more direct way of seeing the integrability of the Klein-Gordon (and indeed, sineGordon) equation with boundary conditions (2.2) is provided by the analysis of [23]. The idea is to show that the method of images can be used on $(-\infty, \infty)$ even in the nonlinear system, so as to replicate the boundary conditions (2.2) on $[0, \infty)$. The scattering of a kink, or anti-kink, from the boundary can be described by a three-soliton solution on $(-\infty, \infty)$. These three solitons consist of the incoming soliton, its mirror image with equal but opposite velocity, and a stationary soliton at the origin (to adjust the boundary conditions). If one takes the infinite rapidity limit of this three-soliton solution then the stationary soliton simply collapses to an overall shift of $\phi$ by a constant, while the mirror images (since they are moving in opposite directions) reduce to a superposition of two wave packets. One thus obtains:

$$
\phi=\phi_{0}+4 \arg \left[1+i \epsilon_{1} e^{-E(\xi-a)}\right]+4 \arg \left[1+i \epsilon_{2} e^{-E(\eta-b)}\right]
$$


where $\xi=\sigma+t, \eta=\sigma-t$. By direct computation one finds that this solution satifies (2.2) with:

$$
e^{E(a+b)}=-\epsilon_{1} \epsilon_{2} \frac{(2 E+g)}{(2 E-g)} .
$$

The constant $\Delta_{B} \equiv-E(a+b)$ represents the delay of the reflected pulse. If one defines the classical boundary scale $\theta_{B}$ via $g=2 \mu e^{-\theta_{B}}$, then this delay may be written as

$$
\Delta_{B}=\log \left(-\epsilon_{1} \epsilon_{2} \tanh \frac{1}{2}\left(\theta-\theta_{B}\right)\right)
$$

Note that the $\operatorname{sign} \epsilon_{2}$ is to be chosen so as to make the argument of the logarithm real. This determines whether the reflection of a kink will be a kink or an anti-kink. Thus we see that $\theta_{B}$ is the scale at which behavior crosses over from the region of the Neumann critical point (where the classical boundary scattering is completely off-diagonal) to the Dirichlet boundary critical point (where classical boundary scattering is diagonal). This result is completely consistent with the infinite-rapidity limit of the delay computed in [23].

Before concluding this section, we wish to observe that the foregoing is not simply a property of some solitonic solutions, but is a general feature of the sine-Gordon equation and its massless limit. The sine-Gordon equation (and any other integrable equation) admits Bäcklund transformations. In particular, consider

$$
\partial_{\eta}(\phi+\psi)=m D \sin \left(\frac{\phi-\psi}{2}\right) ; \quad \partial_{\xi}(\phi-\psi)=\frac{m}{D} \sin \left(\frac{\phi+\psi}{2}\right)
$$

where $D$ is an arbitrary parameter. The point is that $\phi$ satisfies the sine-Gordon equation if and only if $\psi$ does so as well. Taking $m \rightarrow 0$ while keeping $m D$ finite, the Bäcklund transformation becomes:

$$
\partial_{\eta}(\phi+\psi)=m D \sin \left(\frac{\phi-\psi}{2}\right) ; \quad \partial_{\xi}(\phi-\psi)=0
$$

One can easily check that this transformation implies that $\phi$ satisfies the Klein-Gordon equation if and only if $\psi$ does so as well. However, suppose that $\psi$ is a solution to KleinGordon on $[0, \infty)$ satisfying Dirichlet boundary conditions $\phi=\phi_{0}$. It follows immediately from (2.12) that $\psi$ is a solution to Klein-Gordon satisfying (2.2) with $g=-m D$. For any left moving wave-packet, $f(\xi)$, the Dirichlet scattering solution $\phi$ is given by $\phi=\phi_{0}+f(\xi)-$ $f(-\eta)$. The scattering solution, $\psi$, for the same incoming wave-packet reflecting from the boundary conditions (2.2) can then, in principle, be found by by solving (2.12). That is, if one writes $\psi=f(\xi)+r(\eta)$ then $r$ satisfies $r^{\prime}(\eta)=f^{\prime}(\eta)+g \sin \left(\left(r(\eta)+f(-\eta)-\phi_{0}\right) / 2\right)$. 


\section{The quantum $S$ matrix}

We find the quantum $S$ matrix in the same manner as the classical, by taking the massless limit $(G \rightarrow 0)$ of the sine-Gordon $S$ matrix [5]. The Lagrangian for the massive theory is

$$
\mathcal{L}=\frac{1}{2} \int_{0}^{L} d \sigma\left[\left(\partial_{\mu} \phi\right)^{2}+G \cos \beta \phi\right]+g \cos \left[\frac{\beta}{2} \phi(0)\right] .
$$

The physics of the model depends crucially on the parameter $\beta$, which can be scaled out when studying the classical equations of motion. It is convenient to define the parameter

$$
\lambda \equiv \frac{8 \pi}{\beta^{2}}-1 .
$$

In the classical limit $\beta \rightarrow 0$, the results of this section of course reduce to those of the previous section. We should note that a massless $S$ matrix can often be derived directly, without taking a limit of a massive theory [12, 13, 11, 17]. This is done by imposing the constraints of the Yang-Baxter equation, crossing and unitarity.

\subsection{The massless bulk $S$ matrix}

The bulk structure of the quantum sine-Gordon theory is very well known [6, 24, 25]. At any value of $\beta$, the spectrum includes a soliton $S$ and an antisoliton $A$ of mass $m$, with $m \propto G^{(\lambda+1) / 2 \lambda}$. These are the quantized particles corresponding to the classical solitons discussed in the previous section. Moreover, we have bound states (breathers) indexed by $n$ a positive integer less than $\lambda$, with mass $m_{n}=2 m \sin n \pi / 2 \lambda$. As for the classical theory, we obtain the massless limit by sending the rapidities of these particles to $\pm \infty$ and at the same time scaling appropriately the mass $m \rightarrow 0$ so that the energy of each particle remains finite. In this limit we obtain a set of left-moving and right-moving particles. The right movers have dispersion relation $E=P$ and can be parametrized as

$$
E_{S, A}=\mu e^{\theta} \quad E_{n}=2 \mu \sin \left(\frac{n \pi}{2 \lambda}\right) e^{\theta},
$$

where $\mu$ and $\theta$ are now renormalized mass and rapidity. The left movers have $E_{S, A}=$ $-P=\mu e^{-\theta}$ and likewise for the breathers.

The left-left and right-right two-particle $S$ matrices are given by the same formula as in the massive case. The matrices $S_{L L}$ and $S_{R R}$ depend on the difference of renormalized rapidities because the only relativistic invariant that can be formed is the ratio of momenta. (There are no dimensional parameters in the bulk problem). The $S$ matrix is purely 
elastic; individual momenta do not change in a collision. Although the classical $S$ matrix is diagonal, the quantum one is not: the initial state $\left|S\left(\theta_{1}\right) A\left(\theta_{2}\right)\right\rangle$ can scatter to $\left|A\left(\theta_{1}\right) S\left(\theta_{2}\right)\right\rangle$ because $A$ and $S$ have the same mass. For the soliton and antisoliton one has the three usual amplitudes [6]

$$
\begin{aligned}
& a(\theta)=\sin \lambda(\pi+i \theta) Z(\theta) \\
& b(\theta)=-\sin \lambda i \theta Z(\theta) \\
& c(\theta)=\sin \lambda \pi Z(\theta) .
\end{aligned}
$$

where the element $a\left(\theta_{1}-\theta_{2}\right)$ describes the process $\left|S\left(\theta_{1}\right) S\left(\theta_{2}\right)\right\rangle \rightarrow\left|S\left(\theta_{1}\right) S\left(\theta_{2}\right)\right\rangle, b$ describes $S A \rightarrow S A, c$ describes the non-diagonal process $S A \rightarrow A S$, and there is a symmetry under interchange of soliton to antisoliton (corresponding to $\phi \rightarrow-\phi)$. The function $Z(\theta)$ is a well-known normalization factor, which can be written as

$$
Z(\theta)=\frac{1}{\sin \lambda(\pi+i \theta)} \exp \left(i \int_{-\infty}^{\infty} \frac{d y}{2 y} \sin \frac{2 \theta y \lambda}{\pi} \frac{\sinh (\lambda-1) y}{\sinh y \cosh \lambda y}\right)
$$

Notice that when $\lambda$ is an integer, the scattering is diagonal and that $a= \pm b$.

The left-right scattering comes from the $\theta \rightarrow \infty$ limit of (3.3); it is diagonal and rapidity-independent, but this constant phase is different for $S S \rightarrow S S$ and $S A \rightarrow S A$. A non-constant $S_{L R}$ induces a flow between bulk critical points [12, 13, 11]; we will not discuss this here.

The breather-soliton and breather-breather $S$ matrices are well known [6]. The mass-

less limit is taken in the same way, so $S_{L L}$ and $S_{R R}$ are the same as the massive $S$ matrix, while $S_{L R}$ is a constant.

\subsection{The boundary $S$ matrix for solitons}

To study the effect of the dynamical boundary condition we now consider the scattering of these massless particles off the boundary. Since the boundary introduces a scale $T_{B}$ (the "boundary temperature") to the problem, the boundary $S$ matrix element for a particle with momentum $P$ depends on the ratio $P / T_{B}$. Defining the boundary scale $\theta_{B}$ via $T_{B} \equiv \mu e^{-\theta_{B}}$, we see that the $S$ matrix element for a left mover to scatter off the boundary (and thus become a right mover) depends on $\theta-\theta_{B}$.

We can obtain the $S$ matrix from the results of [5]. This $S$ matrix has two free parameters, $\eta$ and $\Theta$. Setting $\phi_{0}=0$ in the boundary potential as we have done corresponds to setting $\eta=0$. The remaining parameter $\Theta$ is a function of the boundary scale $g$. The $S$ matrix then depends on a product of functions of $\alpha, \lambda \alpha+\Theta$, and $\lambda \alpha-\Theta$, where $\alpha$ here is 
the unrenormalized rapidity (which is called $\theta$ in [5]). To get the scattering of left movers off of the boundary, we simply take the limit of very large and negative $\alpha$ and appropriately scale the parameter $\Theta$, so that $\alpha-(\Theta / \lambda) \equiv \theta-\theta_{B}$ remains finite. The effect of this scaling is to obtain left-moving particles of energy comparable to the boundary term energy.

There are two different boundary amplitudes: $P\left(\theta-\theta_{B}\right)$ for the $S \rightarrow S$ and $A \rightarrow A$ processes, and $Q\left(\theta-\theta_{B}\right)$ for $S \rightarrow A$ and $A \rightarrow S$. The symmetry $\phi \rightarrow-\phi$ ensures that the amplitudes are the same when soliton and antisoliton are interchanged. This $S$ matrix is not diagonal; notice that the boundary perturbation breaks the $U(1)$ soliton-number symmetry, thus allowing soliton to scatter into an antisoliton at the boundary. As $g \rightarrow \infty$, this symmetry is restored, so at the Dirichlet fixed point, the element $Q$ must vanish. Taking the limit of the result of [5] as described, one finds

$$
\begin{aligned}
& P(\theta)=e^{\lambda \theta / 2} R(\theta) \\
& Q(\theta)=i e^{-\lambda \theta / 2} R(\theta)
\end{aligned}
$$

where the function $R$ reads

$$
\begin{aligned}
R(\theta) & =\frac{e^{i \gamma}}{2 \cosh \left(\frac{\lambda \theta}{2}-i \frac{\pi}{4}\right)} \prod_{l=0}^{\infty} \frac{Y_{l}(\theta)}{Y_{l}(-\theta)} \\
Y_{l}(\theta) & =\frac{\Gamma\left(\frac{3}{4}+l \lambda-\frac{\lambda i \theta}{2 \pi}\right) \Gamma\left(\frac{1}{4}+(l+1) \lambda-\frac{\lambda i \theta}{2 \pi}\right)}{\Gamma\left(\frac{1}{4}+\left(l+\frac{1}{2}\right) \lambda-\frac{\lambda i \theta}{2 \pi}\right) \Gamma\left(\frac{3}{4}+\left(l+\frac{1}{2}\right) \lambda-\frac{\lambda i \theta}{2 \pi}\right)} .
\end{aligned}
$$

A useful integral representation of $R$ is given by

$$
R(\theta)=\frac{e^{i \gamma}}{2 \cosh \left(\frac{\lambda \theta}{2}-i \frac{\pi}{4}\right)} \exp i \int_{-\infty}^{\infty} \frac{d y}{2 y} \sin \frac{2 \lambda \theta y}{\pi} \frac{\sinh (\lambda-1) y}{\sinh 2 y \cosh \lambda y}
$$

The constant phase $\gamma$ depends on $\lambda$ and turns out to be necessary to satisfy the crossunitarity relation [5]. For right movers scattering off a boundary, the answer is similar but differs in some constant phases and signs.

We can check this answer in several limits. As $\theta_{B} \rightarrow \infty$, we obtain the Neumann fixed point. Since $P\left(\theta-\theta_{B}\right) \rightarrow 0$ in this limit, the boundary scattering is completely offdiagonal, so that the $U(1)$ soliton-number symmetry is maximally violated. As $\theta_{B} \rightarrow-\infty$, we reach the Dirichlet fixed point, where the scattering is diagonal. The results at both limits follow from the analysis of [2].

We can also check the answer at several values of $\lambda$. The case $\lambda=1$, where the perturbing operator has dimension $\frac{1}{2}$, has already been solved [16]. It was shown explicitly 
in the corresponding lattice model that the bulk theory is equivalent to two critical Ising models, and that the boundary interaction corresponds to a boundary magnetic field in one of the two Ising models. We have the same result here. The bulk theory corresponds to a free massless Dirac fermion, where the soliton corresponds to the fermion and the antisoliton the antifermion. This is easily seen in (3.3): the $S$ matrix elements are $a=b=$ $1, c=0$. We can decompose this into the massless Majorana fermions of two critical Ising models by looking at the linear combinations $(|S\rangle+|A\rangle)$ and $(|S\rangle-|A\rangle)$. The corresponding boundary $S$ matrix elements are then $P+Q$ and $P-Q$. For $\lambda=1$, we then have

$$
\begin{aligned}
& P+Q=1 \\
& P-Q=\tanh \left(\frac{\theta}{2}-\frac{i \pi}{4}\right)
\end{aligned}
$$

The $S$ matrix for an Ising model in a boundary magnetic field indeed is $\tanh \left(\frac{\theta}{2}-\frac{i \pi}{4}\right)$, as can be seen by explicit calculation or by taking the limit of the massive $S$ matrix in [5].

Another interesting value is $\lambda=0$, where the dimension of the perturbing operator is 1 , so that the operators $\exp (i 4 \pi \phi), \exp (-i 4 \pi \phi)$ and $\partial \phi$ are the generators of an $\mathrm{SU}(2)$ symmetry at the boundary. The fact that $\cos 4 \pi \phi$ can be rotated into $\partial \phi$ means that the operator is exactly marginal, and the boundary interaction does not break the conformal symmetry. The resulting boundary $S$ matrix cannot depend on rapidity: because there are no dimensional parameters in the theory, there is no way of making a relativistic invariant out of a single momentum. Indeed, we see that the rapidity dependence in the $S$ matrix (3.4) goes away. This does not mean that the $S$ matrix is trivial since we can scale $\theta_{B}$ with $\lambda$. Actually, in the limit $\lambda \rightarrow 0$ this is necessary to get a finite value of $g$, following the relation of $g$ and $\theta_{B}$ (see (4.16) below). Returning to the original parameter $\Theta$, we have

$$
\begin{aligned}
& P=\frac{e^{\Theta / 2}}{\sqrt{2 \cosh \Theta}} \\
& Q=i \frac{e^{-\Theta / 2}}{\sqrt{2 \cosh \Theta}} .
\end{aligned}
$$

This is now a simple rotation in the $S, A$ space

$$
\left(\begin{array}{cc}
\cos \pi g_{R} & i \sin \pi g_{R} \\
i \sin \pi g_{R} & \cos \pi g_{R}
\end{array}\right)
$$

with

$$
\tan \pi g_{R}=e^{-\Theta}
$$

This is exactly the picture obtained in [26,27,28], where $g_{R}$ is the renormalized coupling constant. 


\subsection{The boundary $S$ matrix for breathers}

One can easily treat similarly the boundary $S$ matrix for the $n$th breather [29]. The integrability requires that it be diagonal. One finds amplitudes that depend on the parity of $n$ :

$$
\frac{1}{i} \frac{d}{d \theta} \ln R^{(2 k)}(\theta)=2 \sum_{l=1}^{k} \frac{\cosh \theta \cos (l-1 / 2) \pi / \lambda}{\cosh ^{2} \theta-\sin ^{2}(l-1 / 2) \pi / \lambda} .
$$

and

$$
\frac{1}{i} \frac{d}{d \theta} \ln R^{(2 k-1)}(\theta)=\frac{1}{\cosh \theta}+2 \sum_{l=1}^{k-1} \frac{\cosh \theta \cos l \pi / \lambda}{\cosh ^{2} \theta-\sin ^{2} l \pi / \lambda} .
$$

The integral representation is

$$
R^{(n)}(\theta)=\exp i \int_{-\infty}^{\infty} \frac{d y}{2 y} \sin \frac{2 \lambda \theta y}{\pi} \frac{\sinh n y}{\sinh y \cosh \lambda y} .
$$

\section{The exact boundary free energy}

Using this scattering description we can now compute the evolution of the boundary entropy between Neumann and Dirichlet boundary conditions. Recall that if we consider the theory defined by the Lagrangian (1.1) at temperature $T$ the free energy reads

$$
F=f L-T \ln \left(g_{1} g_{2}\right)
$$

where the bulk term $f$ is $L$ independent, $\ln g_{1}$ and $\ln g_{2}$ are boundary entropies associated with the two boundaries at $\sigma=0, L$. The boundary interaction does not affect $f$, but it causes $g_{1}$ to flow from its Neumann value to its Dirichlet value. As we will see, $g_{1}$ decreases, in accordance with the " $g$-conjecture" of [30]. In this section, we explicitly compute $g_{1}$ as a function of the boundary scale by using the thermodynamic Bethe ansatz (TBA) [9, 10].

\subsection{The TBA equations in the presence of a boundary}

The TBA exploits the fact in an integrable model, we can find the exact relation between the density of states $P$ and the particle density $\rho$. (Often the quantity $P-\rho$ is called the density of holes $\rho^{h}$.) In models that are not integrable, one generally uses the free-particle density of states and then uses perturbation theory in the interaction parameters. However, the fact that the scattering is completely elastic and factorizable in an integrable model gives an exact functional relation between $P$ and $\rho$, known as the 
Bethe equation. We then write down the free energy in terms of $P$ and $\rho$. The particle density $\rho$ of the system at temperature $T$ is then found by minimizing the free energy, using the relation of $P$ to $\rho$.

We discuss the case when the bulk and boundary $S$ matrices are diagonal. When there are $p$ different species of particle, we have the two-particle $S$ matrix elements $S_{r s}\left(\theta_{1}-\theta_{2}\right)$, where $r$ and $s$ run from 1 to $p$. These $S$ matrix elements are the phase shift in the wavefunction when two particles are exchanged. We also have the boundary $S$ matrix elements $R_{r}\left(\theta-\theta_{B}\right)$, which gives the phase shift when a particle of species $r$ bounces off a wall and changes its rapidity from $\theta$ to $-\theta$. We have a gas of $\mathcal{N}$ particles on a line of length $L$, with the $i$ th particle of species $r_{i}$. As in the Kondo problem [17], we map the problem onto a line of length $2 L(-L<\sigma<L)$ by considering the right movers to be left movers with $\sigma<0$. Thus we have only left movers scattering among themselves and off of the boundary, which can now be thought of as an impurity (a particle with rapidity $\theta_{B}$ ). (This trick is common to boundary conformal field theory and can only be used in the massless limit.) For simplicity, we put periodic boundary conditions on the system; these do not change the boundary effects at $\sigma=0$.

First we write the Bethe equations. These equations quantize the set of allowed rapidities for a system of left-moving particles on a circle of length $2 L$ with an impurity at $\sigma=0$. They are obtained by collecting all the phase shifts due to particle-particle and particle-impurity scattering when arguments in the wave functions are analytically continued $\sigma \rightarrow \sigma+2 L$. Demanding that the wavefunction be periodic gives the constraint

$$
e^{-2 i \mu_{r_{i}} \exp \theta_{i} L} \prod_{j=1, j \neq i}^{\mathcal{N}} S_{r_{i} r_{j}}\left(\theta_{i}-\theta_{j}\right) R_{r_{i}}\left(\theta_{i}-\theta_{B}\right)=1,
$$

where $E=-P=\mu_{r} \exp (-\theta)$ for a particle of type $r$. Since the scattering is diagonal, we can define the densities $\rho_{r}$ and $\rho_{r}^{h}$ for each species of particle, so that $\left(\rho_{r}(\theta)+\rho_{r}^{h}(\theta)\right) 2 L d \theta$ gives the number of allowed rapidities between $\theta$ and $\theta+d \theta$ for species $r$, and $\rho_{r}$ gives the density of filled states. Taking the derivative of the logarithm of (4.2) gives one equation for every type of particle:

$$
2 \pi\left(\rho_{r}(\theta)+\rho_{r}^{h}(\theta)\right)=\mu_{r} e^{-\theta}+\sum_{s=1}^{p} \varphi_{r s} \star \rho_{s}(\theta)+\frac{1}{2 L} \kappa_{r}\left(\theta-\theta_{B}\right),
$$

where $\star$ denotes convolution:

$$
f \star g(\alpha) \equiv \int_{-\infty}^{\infty} d \alpha^{\prime} f\left(\alpha-\alpha^{\prime}\right) g\left(\alpha^{\prime}\right)
$$


and

$$
\begin{aligned}
\varphi_{r s}(\theta) & =-i \frac{d}{d \theta} \ln S_{r s}(\theta) \\
\kappa_{r}\left(\theta-\theta_{B}\right) & =-i \frac{d}{d \theta} \ln R_{r}\left(\theta-\theta_{B}\right) .
\end{aligned}
$$

The effect of the boundary is seen in the last piece of (4.3), proportional to $1 / L$.

We now consider the free energy $\mathcal{F}=\mathcal{E}-T \mathcal{S}$. For the energy we have

$$
\mathcal{E}=2 L \int d \theta \sum_{r=1}^{p} \rho_{r}(\theta) \mu_{r} e^{-\theta}
$$

and entropy

$$
\mathcal{S}=2 L \int d \theta \sum_{r=1}^{p}\left[\left(\rho_{r}+\rho_{r}^{h}\right) \ln \left(\rho_{r}+\rho_{r}^{h}\right)-\rho_{r} \ln \rho_{r}-\rho_{r}^{h} \ln \rho_{r}^{h}\right]
$$

(The particles act like fermions when filling levels.) The free energy is the value of $-T \ln Z$ at the saddle point of the partition sum or path integral: as a function of $\rho, \mathcal{F}$ is at a miminum. Via

$$
\frac{d \mathcal{F}}{d \rho_{r}}=\frac{\partial \mathcal{F}}{\partial \rho_{r}}+\sum_{s} \frac{\partial \mathcal{F}}{\partial \rho_{s}^{h}} \frac{\partial \rho_{s}^{h}}{\partial \rho_{r}}=0
$$

one obtains equations for densities, using (4.3) to determine $\partial \rho_{s}^{h} / \partial \rho_{r}$. These equations do not depend on the boundary term, because boundary terms only appear in the Bethe equations, and disappear in a variation of the densities. Defining

$$
e^{\bar{\epsilon}_{r}(\theta)} \equiv \frac{\rho_{r}^{h}(\theta)}{\rho_{r}(\theta)},
$$

one finds the well-known equations [9, 10]

$$
\epsilon_{r}(\theta)=\frac{\mu_{r}}{\mu} e^{-\theta}-\frac{1}{2 \pi} \sum_{s=1}^{p} \varphi_{r s} \star \ln \left(1+e^{-\epsilon_{s}}\right),
$$

where $\epsilon(\theta)=\bar{\epsilon}(\theta+\ln (\mu / T))$. Boundary terms enter when expressing the free energy in terms of the $\epsilon_{r}$. We find them by rewriting the energy (4.5) as

$$
\begin{aligned}
\mathcal{E} & =2 L \int \sum_{r=1}^{p} \rho_{r}\left[T \bar{\epsilon}_{r}+\frac{T}{2 \pi} \sum_{s=1}^{p} \varphi_{r s} \star \ln \left(1+e^{-\bar{\epsilon}_{s}}\right)\right] \\
& =2 L T \int\left[\sum_{r=1}^{p} \rho_{r} \bar{\epsilon}_{r}+\sum_{s=1}^{p}\left(\rho_{s}+\rho_{s}^{h}-\frac{\mu_{s}}{2 \pi} e^{-\theta}-\frac{1}{4 \pi L} \kappa_{s}\right) \ln \left(1+e^{-\bar{\epsilon}_{s}}\right)\right] \\
& =T \mathcal{S}-\int \sum_{r=1}^{p}\left[\frac{T L \mu_{r}}{\pi} e^{-\theta}+\frac{T}{2 \pi} \kappa_{r}\right] \ln \left(1+e^{-\bar{\epsilon}_{r}}\right)
\end{aligned}
$$


where we used (4.7) to get to the first line, (4.3) to get to the second, and (4.6) to get to the third. Thus we have

$$
\mathcal{F}=\mathcal{F}_{\text {bulk }}-T \int \frac{d \theta}{2 \pi} \sum_{r=1}^{p} \kappa_{r}\left(\theta-\ln \left(T / T_{B}\right)\right) \ln \left(1+e^{-\epsilon_{r}(\theta)}\right) .
$$

It is a general result that $\mathcal{F}_{b u l k}=-\frac{\pi c}{6} T^{2} L$ in a massless bulk theory, where $c$ is the central charge of the conformal field theory 31.

Although the equations (4.7) for $\epsilon(\theta)$ cannot be solved explicitly for all temperatures, the free energy is easy to evaluate as $T \rightarrow 0$ and $T \rightarrow \infty$, as we will show in sect. 4.3. Moreover, one can extract the analytic values of critical exponents by looking at the form of the expansions around these fixed points. Also, they are straightforward to solve numerically for any $T$.

Several notes of caution are necessary. At the order we are working, the formula (4.6) for the entropy is not quite correct, because there are $1 / L$ corrections to the Stirling formula used in its derivation. Also, at this order, the logarithm of the partition function is not $\mathcal{E}-T \mathcal{S}$ : it depends not only on the saddle point value of the sum over all states, but also on fluctuations. Their net effect is that we cannot compute the $g$ factors from $\mathcal{F}$ alone. However, both of these corrections are subleading contributions to the bulk free energy, and do not depend on the boundary conditions. Therefore we can still compute differences of $g$ factors from $\mathcal{F}$; the corrections are independent of the boundary scale $\theta_{B}$ and cancel out of the difference [32].

\subsection{The exact boundary free energy for $\lambda$ integer}

In this section we consider only the case $\lambda$ a positive integer. At these values, a major simplification takes place: the bulk scattering (3.3) is diagonal. The boundary scattering still is not, but we can redefine our states to be $| \pm\rangle \equiv(|S\rangle \pm|A\rangle) / \sqrt{2}$ as in the previous section, so that the boundary scattering is now diagonal: 1

$$
\begin{aligned}
& R^{+}(\theta)=P+Q=\cosh \left(\frac{\lambda \theta}{2}-\frac{i \pi}{4}\right) e^{i \pi / 4} R(\theta) \\
& R^{-}(\theta)=P-Q=\cosh \left(\frac{\lambda \theta}{2}+\frac{i \pi}{4}\right) e^{-i \pi / 4} R(\theta)
\end{aligned}
$$

${ }^{1}$ If $\lambda$ is even, this actually makes the bulk scattering completely off-diagonal (e.g. $|++\rangle$ scatters to $|--\rangle$ ), but the TBA equations turn out the same. 
Using the analysis of the previous subsection, we can read off the answer. There are $\lambda+1$ particles: the breathers, which we index $n=1 \ldots \lambda-1$, and the \pm particles. We define the Fourier transform as

$$
\tilde{f}(y)=\int_{-\infty}^{\infty} \frac{d \theta}{2 \pi} e^{i 2 \lambda \theta y / \pi} f(\theta) .
$$

The bulk kernels $\phi$ are well known [33]; they can be written in the form

$$
\begin{aligned}
& \tilde{\varphi}_{n l}=\delta_{n l}-2 \frac{\cosh y \cosh (\lambda-n) y \sinh l y}{\cosh \lambda y \sinh y} \quad n, l=1 \ldots \lambda-1 ; n \geq l \\
& \tilde{\varphi}_{n, \pm}=-\frac{\cosh y \sinh n y}{\cosh \lambda y \sinh y} \\
& \tilde{\varphi}_{ \pm, \pm}=\tilde{\varphi}_{ \pm, \mp}=-\frac{\sinh (\lambda-1) y}{2 \cosh \lambda y \sinh y},
\end{aligned}
$$

with $\varphi_{r s}=\varphi_{s r}$. The boundary kernels follow from (4.4), and are

$$
\begin{aligned}
\tilde{\kappa}_{n} & =\frac{\sinh n y}{2 \sinh y \cosh \lambda y} \\
\tilde{\kappa}_{-} & =\frac{\sinh (\lambda-1) y}{2 \sinh 2 y \cosh \lambda y}+\frac{1}{2 \cosh y} \\
\tilde{\kappa}_{+} & =\frac{\sinh (\lambda-1) y}{2 \sinh 2 y \cosh \lambda y} .
\end{aligned}
$$

The equations for the $\epsilon_{r}$ can be written in a much simpler form by using a few trigonometric indentities. One finds

$$
\epsilon_{r}=K \star \sum_{s} N_{r s} \ln \left(1+e^{\epsilon_{s}}\right),
$$

where the label $s$ runs over breathers and \pm , the kernel $K(\theta)=1 / 2 \pi \cosh \theta$, and $N_{r s}$ is the incidence matrix of the following diagram

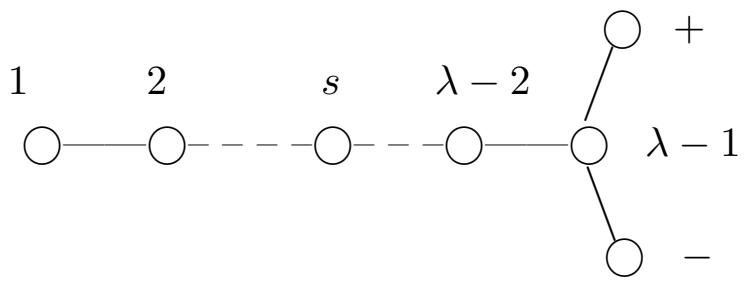

The dependences on the mass ratios seems to have disappeared from (4.11), but they appear as an asymptotic condition: the original equations (4.7) indicates that the solution must satisfy

$$
\epsilon_{r} \rightarrow \frac{\mu_{r}}{\mu} e^{-\theta} \quad \text { as } \theta \rightarrow-\infty .
$$


To recapitulate: the impurity contribution to the free energy is

$$
\left.\mathcal{F}_{i m p}=-T \int \frac{d \theta}{2 \pi} \sum_{r} \kappa_{r}\left(\theta-\ln \left(T / T_{B}\right)\right) \ln \left(1+e^{-\epsilon_{r}(\theta)}\right)\right)
$$

where the $\kappa_{r}$ are given by (4.10), and the $\epsilon_{r}$ are the solutions of the non-linear integral equations (4.11).

At non-integer values of $\lambda$, the bulk $S$ matrix is not diagonal. As a result both bulk and boundary scattering have to be simultaneously diagonalized. It is known how to do this in the bulk [34], and the inclusion of the boundary can be presumably be acomplished by using the quantum inverse scattering method along the lines of [35] and [32]. The simplest values to do should be $\lambda=1 / t$ with $t$ integer, where there are no breathers, and the bulk TBA system is given by (4.11) with different conditions for $\theta \rightarrow-\infty$. In fact, using the ideas of 32 and the "duality" of TBA equations for $\lambda=t$ and $\lambda=\frac{1}{t}$ we have been able to conjecture a TBA for the latter case that reproduces all expected results for our flow. For simplicity we do not present this TBA here.

\subsection{The Neumann and Dirichlet limits}

We can evaluate the impurity free energy explicitly in several limits. In the IR limit $T / T_{B} \rightarrow 0$ the integral is dominated by $\theta \rightarrow-\infty$ where the source terms in (4.7) become very big. Hence $\epsilon_{r}(-\infty)=\infty$ and the impurity free energy vanishes in this limit. In the UV limit $T / T_{B} \rightarrow \infty$ the integrals are dominated by the region where $\theta$ is large so that the source terms disappear in (4.7) and the $\epsilon_{r}$ go to constants:

$$
x_{n} \equiv e^{\epsilon_{n}(\infty)}=(n+1)^{2}-1 ; \quad x_{ \pm}=\lambda .
$$

Therefore we obtain

$$
\begin{aligned}
\ln \frac{g_{N}}{g_{D}} & =\left.\frac{-\mathcal{F}_{i m p}}{T}\right|_{U V}-\left.\frac{-\mathcal{F}_{i m p}}{T}\right|_{I R} \\
& =\sum_{n=1}^{t-2} I^{(n)} \ln \left(1+1 / x_{n}\right)+\left(I^{(+)}+I^{(-)}\right) \ln \left(1+1 / x_{ \pm}\right)
\end{aligned}
$$

where

$$
I^{(r)} \equiv \int \frac{d \theta}{2 \pi} \kappa_{r}(\theta)=\tilde{\kappa}(0)
$$


Thus $I^{(n)}=n / 2$ and $I^{(+)}+I^{(-)}=\lambda / 2$, and we find

$$
\begin{aligned}
\ln \frac{g_{N}}{g_{D}} & =\frac{\lambda}{2} \ln \frac{\lambda+1}{\lambda}+\sum_{n=1}^{\lambda-1} \frac{n}{4} \ln \frac{(n+1)^{2}}{n(n+2)} \\
& =\frac{1}{2} \ln (\lambda+1) .
\end{aligned}
$$

This is agreement with the ratio calculated from conformal field theory, as detailed in the Appendix.

We can also find the dimension of the perturbing operators. From the equations (4.7) one deduces [36] the following expansions for $T / T_{B}$ large:

$$
Y_{r}(\theta)=e^{\epsilon_{r}(\theta)}=\sum_{j} Y_{r}^{(j)} e^{-2 j \lambda \theta /(\lambda+1)}
$$

As a result it is straightforward to see that near $g=0, \mathcal{F}$ can be expanded in powers of $\left(T_{B} / T\right)^{2 \lambda /(\lambda+1)}$. On the other hand we expect $\mathcal{F}$ to be an analytic function of $g^{2}$. Hence

$$
g \propto\left(\mu e^{-\theta_{B}}\right)^{\lambda /(\lambda+1)}
$$

This agrees with the conformal result that the perturbing operator $\cos [\beta \phi(0) / 2]$ has boundary dimension $x=1 /(\lambda+1)=\beta^{2} / 8 \pi$. In the IR limit of $T / T_{B}$ small, one can expand out the kernels $\kappa_{r}$ in powers of $\exp \left(\theta_{b}-\theta\right)$. This leads to the fact that the irrelevant operator which perturbs the Dirichlet boundary conditions has dimension $x=2$. This is the energy-momentum tensor. (We note that there is another irrelevant operator in the spectrum with dimension $x=\lambda+1$, which for $0 \leq \lambda<1$ is the appropriate perturbing operator [2].)

\section{Future directions}

We see that at $\lambda=0$, the boundary $S$ matrix is a constant for all $\theta$. This is expected, since we can continuously interpolate from Neumann to Dirichlet without destroying the conformal symmetry. Using the conformal symmetry, one in fact is able to calculate the full partition function along this interpolation 26-28], not just at the Neumann and Dirichlet points as in the Appendix. It would be interesting to precisely understand how to map the scattering basis discussed in [27] to the scattering basis used here. This would provide a great deal of insight into the long-confusing relation between the states arising from the 
Bethe Ansatz to those in the Virasoro towers of a conformal field theory (see [37], for example).

The quantum $S$ matrix for Dirichlet boundary conditions $\phi(0)=\phi_{0}$ can be derived explicitly by applying the Bethe ansatz to an underlying lattice model, the XXZ spin chain with a boundary magnetic field [32]. Presumably the more general $S$ matrix studied here corresponds to the XXZ chain with boundary terms of the type $\sigma^{ \pm}$that break the $U(1)$ symmetry [38].

By expanding the partition function of our system in powers of $g$ we obtain a onedimensional neutral Coulomb gas of particles on a circle, whose solution is therefore indirectly provided by the TBA. Previously, such a Coulomb gas had been solved for only one type of charge.

Perhaps the most interesting future direction is the application of these results to the experimentally realizable system of tunnelling between fractional quantum-Hall edges [39]. We will report on this application soon [40].

\section{Acknowledgements:}

We thank the many participants at the conference SMQFT 94 for discussions. This work was supported by the Packard Foundation, the National Young Investigator program (NSF-PHY-9357207) and the DOE (DE-FG03-84ER40168).

\section{Appendix A. Neumann and Dirichlet partition functions}

We here use standard conformal theory techniques to calculate the partition function of our quantum system on a cylinder of length $L$ and circumference $1 / T$ and Neumann or Dirichlet boundary conditions at the ends.

With Dirichlet boundary conditions on both sides, winding modes are not allowed around the cylinder, but they are allowed along it. Thus the field $\phi$ obeys $\phi(\sigma=0)=$ $\phi(\sigma=L)+n 4 \pi / \beta$ with $n$ an integer. (In the usual conventions, this corresponds to a bosonic radius of $\sqrt{4 \pi} / \beta$.) Hence 41

$$
Z^{D D}=\frac{1}{\eta(w)} \sum w^{8 \pi n^{2} / \beta^{2}}
$$

where $w=e^{-\pi / L T}$. After modular transformation one finds

$$
Z^{D D}=\left(\frac{\beta^{2}}{16 \pi}\right)^{1 / 2} \frac{1}{\eta\left(q^{2}\right)} \sum q^{\beta^{2} n^{2} / 16 \pi},
$$


where $q=e^{-2 \pi L T}$. With Neumann on one side and Dirichlet on the other, no winding modes of any sort are allowed. The energy, however, is quantized with half-integer eigenvalues. Using the Jacobi triple product formula gives

$$
Z^{N D}=\frac{1}{\eta(w)} \sum w^{(n-1 / 2)^{2} / 4}
$$

which after modular transformation is

$$
Z^{N D}=\frac{1}{\sqrt{2} \eta\left(q^{2}\right)} \sum(-1)^{n} q^{2 n^{2}}
$$

By taking the ratio in the limit of large $L$, we find

$$
\frac{g_{D}}{g_{N}}=\left(\frac{\beta^{2}}{8 \pi}\right)^{1 / 2} \text {. }
$$

in agreement with (4.15). 


\section{References}

[1] N. Andrei, K. Furuya, and J. Lowenstein, Rev. Mod. Phys. 55 (1983) 331;

A.M. Tsvelick and P.B. Wiegmann, Adv. Phys. 32 (1983) 453.

[2] C.L. Kane and M.P.A. Fisher, Phys. Rev. B46 (1992) 15233.

[3] A. Schmid, Phys. Rev. Lett. 51 (1983) 1506;

F. Guinea, V. Hakim and A. Muramatsu, Phys. Rev. Lett. 54 (1985) 263.

[4] J. Cardy, Nucl. Phys. B240 (1984) 514.

[5] S. Ghoshal and A.B. Zamolodchikov, "Boundary State and Boundary $S$ Matrix in Two-Dimensional Integrable Field Theory", RU-93-20, hep-th/9306002.

[6] A.B. Zamolodchikov and Al.B. Zamolodchikov, Ann. Phys. 120 (1979) 253.

[7] A.B. Zamolodchikov, Adv. Stud. Pure Math. 19 (1989) 1.

[8] G. Mussardo, Phys. Rep. 218 (1992) 215.

[9] C.N. Yang and C.P. Yang, J. Math. Phys. 10 (1969) 1115.

[10] Al.B. Zamolodchikov, Nucl. Phys. B342 (1991) 695.

[11] P. Fendley and H. Saleur, "Massless integrable quantum field theories and massless scattering in 1+1 dimensions", USC-93-022, hep-th/9310058.

[12] Al.B. Zamolodchikov, Nucl. Phys. B358 (1991) 524.

[13] A.B. Zamolodchikov and Al.B. Zamolodchikov, Nucl. Phys. B379 (1992) 602.

[14] L.D. Faddeev and L.A. Takhtajan, Phys. Lett. 85A (1981) 375.

[15] N.Yu Reshetikhin and H. Saleur, "Lattice regularization of massive and massless integrable field theories", USC-93-020, hep-th/9309135.

[16] F. Guinea, Phys. Rev. B32 (1985) 7518.

[17] P. Fendley, Phys. Rev. Lett. 71 (1993) 2485, cond-mat/9304031.

[18] S. Dasmahapatra, R. Kedem, T.R. Klassen, B.M. McCoy and E. Melzer, "Quasiparticles, Conformal Field Theory, and q-series", hep-th/9303013.

[19] M. Ablowitz and H. Segur, Solitons and the inverse scattering transform, Philadelphia: SIAM, 1981

[20] R. Sasaki and I. Yamanaka, Adv. Stud. in Pure Math. 16 (1988) 271.

[21] R. Jackiw and G. Woo, Phys. Rev. D12 (1975) 1643.

[22] V.E. Korepin, Th. Math. Phys. 34 (1978) 1.

[23] H. Saleur, S. Skorik and N.P. Warner, in preparation.

[24] L. Faddeev, E. Sklyanin and L. Takhtajan, Th. Math. Phys. 40 (1979) 688.

[25] V.E. Korepin, Th. Math. Phys. 41 (1979) 953.

[26] C.G. Callan and I.R. Klebanov, Phys. Rev. Lett. 72 (1994) 1968, hep-th/9311092.

[27] C.G. Callan, I. Klebanov, A.W.W. Ludwig and J. Maldacena, "Exact solution of a boundary conformal field theory", PUPT-1450, IASSNS-HEP-94/15, hep-th/9402113.

[28] J. Polchinski and L. Thorlacius, "Free fermion representation of a boundary conformal field theory", NSF-ITP-94-29, hep-th/9404008. 
[29] S. Ghoshal, "Bound State Boundary $S$ Matrix of the Sine-Gordon Model", RU-93-51, hep-th/9310188.

[30] I. Affleck and A. Ludwig, Phys. Rev. Lett. 67 (1991) 161.

[31] H.W. Blote, J.L. Cardy and M.P. Nightingale, Phys. Rev. Lett. 56 (1986) 742; I. Affleck, Phys. Rev. Lett. 56 (1980) 746.

[32] P. Fendley and H. Saleur, "Deriving Boundary $S$ Matrices", hep-th/9402045, to appear in Nucl. Phys. B.

[33] T.R. Klassen and E. Melzer, Nucl. Phys. B338 (1990) 485.

[34] M. Takahashi and M. Suzuki, Prog. Th. Phys. 48 (1972) 2187.

[35] E.K. Sklyanin, J.Phys. A21 (1988) 2375.

[36] Al.B. Zamolodchikov, Phys. Lett. B253 (1991) 391.

[37] E. Melzer, "Massive-Conformal Dictionary", TAUP-2109-93, hep-th/9311058.

[38] H.J. de Vega and A. Gonzalez Ruiz, "Boundary K matrices for the XYZ, XXZ and XXX spin chains", LPTHE-93-29, hep-th/9306089.

[39] X.G. Wen, Phys. Rev. B41 (1990) 12838; K. Moon, H. Yi, C.L. Kane, S.M. Girvin, and M.P.A. Fisher, Phys. Rev. Lett. 71 (1993) 4381.

[40] P. Fendley, A. Ludwig and H. Saleur, in preparation.

[41] S. Eggert and I. Affleck, Phys. Rev. B46 (1993) 10866. 